\title{
Superconductivity in Ca-intercalated bilayer graphene
}

\author{
I.I. Mazin ${ }^{1}$ and A. V. Balatsky ${ }^{2,3, *}$ \\ ${ }^{1}$ Code 6393, Naval Research Laboratory, Washington, D.C. 20375 \\ ${ }^{2}$ Theoretical Division, Los Alamos National Laboratory, Los Alamos, New Mexico 87545, USA \\ ${ }^{3}$ Center for Integrated Nanotechnology, Los Alamos National Laboratory, Los Alamos, New Mexico 87545, USA
}

(Dated: Printed November 7, 2018)

\begin{abstract}
Recent observation of proximity effect [1] has ignited interest in supercondcutivity in graphene and its derivatives. We consider Ca-intercalated graphene bilayer and argue that it is a superconductor, and likely with a sizeable $T_{c}$. We find substantial and suggestive similarities between Ca-intercalated bilayer $\left(\mathrm{C}_{6} \mathrm{CaC}_{6}\right)$, and $\mathrm{CaC}_{6}$, an established superconductor with $T_{c}=11.5 \mathrm{~K}$. In particular, the nearly free electron band, proven to be instrumental for superconductivity in intercalated graphites, does cross the chemical potential in $\left(\mathrm{C}_{6} \mathrm{CaC}_{6}\right)$, despite the twice smaller doping level, satisfying the so-called "Cambridge criterion". Calculated properties of zone-center phonons are very similar to those of $\mathrm{CaC}_{6}$. This suggests that the critical temeperature would probably be on the same scale as in $\mathrm{CaC}_{6}$.
\end{abstract}

PACS numbers: Pacs Numbers:

The graphite becomes superconducting after intercalation with alkali elements, with the transition temperature ranging from below $1 \mathrm{~K}$ for $\mathrm{KC}_{8}$ to $11.5 \mathrm{~K}$ for $\mathrm{CaC}_{6}$ $[2,[3,4,[5]$. Mechanism of superconductivity seems to be consistent with phonon mediated pairing [6]. These graphite intercalated compounds (GIC) open a promising route to an alternative class of superconducting of materials with tunable properties.

The recent discovery of graphene 7, 8], a single sheet of carbon atoms, has naturally raised a question of superconductivity in graphene [9]. Proximity induced superconductivity in pure graphene has been demonstrated 1]. This work shows the potential for developing new superconducting devices starting with $2 \mathrm{D}$ graphene as a basis material.

The purpose of this Letter is to investigate routes of doping graphene with $\mathrm{Ca}$ to an extent that would induce intrinsic superconductivity. In doing that, we will heavily rely on insights from now well understood superconductivity in intercalated graphites.

Obviously, for a large number of graphene layers we would have to recover bulk superconductivity of $\mathrm{CaC}_{6}$. Hence, if superconducting state is indeed possible, one can intercalate multilayer compounds with intermediate numbers of layers and investigate the crossover between $2 \mathrm{D}$ superconducting state in a few layers and the increasingly 3D character of multilayer compositions. This bottom up approach leads to a reasonable question to ask: is an intercalated graphene bilayer a superconductor in itself? We will argue that the answer to this question is affirmative.

Let us recall the basics of superconductivity in $\mathrm{CaC}_{6} \cdot$ [6] It was proposed at a very early stage that (i) soft Ca modes contribute substantially to electronphonon coupling [11] and (ii) a nearly free-electron threedimensional electronic band, " $\zeta$ ", an analogue of the free electron $s$-band in Ca metal, plays an indispensable role

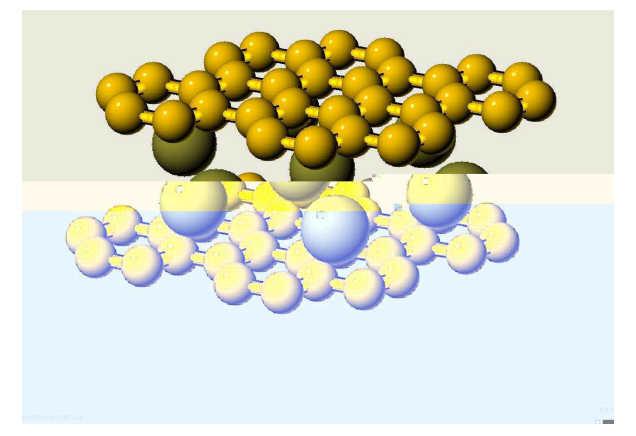

Figure 1: Crystal structure of Ca C6 bilayer is shown. $\mathrm{Ca}$ atom sits in the approximately in the middle of one of hexagons of $C_{6}$.

in superconductivity[10]. An important observation was made by Littlewood and collaborators [10] who pointed out that for all known superconducting GIC a nearly free electron band (which is well above the Fermi level in the pure graphite) crosses the Fermi level (the "Cambridge criterion"). Detailed calculations confirmed both conjectures [12, 13] and found that the Ca phonons provide about half of the coupling strength [14], while electrons experiencing the strongest pairing interaction are those in the nearly free electron band, although the carbon electrons also show a sizeable coupling with phonons.

This naturally suggests $\mathrm{Ca}$ as a dopant for graphene. We focus on the bilayer graphene case as a first compound that can be truly intercalated. [15]. Moreover, intercalation with $\mathrm{Ca}$ should provide additional rigidity, making the new superconductor structurally robust. It is not obvious, however, that intercalating a bilayer will be as effective as intercalating the bulk graphite. As a very minimum, intercalating a bilayer to the same degree as graphite provides twice less carriers, e.g., $\mathrm{C}_{6} \mathrm{CaC}_{6}$ vs. $\mathrm{CaC}_{6}$. [16]. Therefore, the first question to ask is 
whether the emprirical "Cambridge rule" holds, that is, whether the Ca-derived nearly-free-electron band does cross the Fermi level? Note that as opposed to the graphites, where this band is three-dimensional and its density of states depends on filling, in bilayer graphene it is $2 \mathrm{D}$ and therefore has filling-independent density of states (as long as it crosses the Fermi level). The other point is to investigate how similar are the elastic properties of Ca intercalated between two graphene sheets to that of the Ca in graphite. In what follows we will answer both questions affirmatively, based on first principle calculations, and will therefore suggest that Ca-intercalated bilayer graphene should be a superconductor with a critical temperature comparable to that of $\mathrm{CaC}_{6}$.

To this end, we have used the standard full-potential linear augmented wave method for band structure calculations 17] in conjunction with the density functional theory in a generalized gradient approximation. The setup and technical details have been described elsewhere 11. To imitate an isolated $\mathrm{C}_{6} \mathrm{CaC}_{6}$ trilayer we used an $18.2 \AA$ thick slab and optimized the distance between the $\mathrm{C}$ layers. The latter came out to be 4.64 $\AA$, only slightly expanded compared to that in the bulk $\mathrm{CaC}_{6}$, namely. This, by itself, indicates that bonding is similar and the phonon property will probably also be similar. To verify that latter conjecture we have performed frozen phonon calculations of a $\mathrm{Ca} E_{1 u}$ phonon mode, corresponding to the Ca layer sliding with respect to the $\mathrm{C}$ layers. The results are shown in Fig. 2

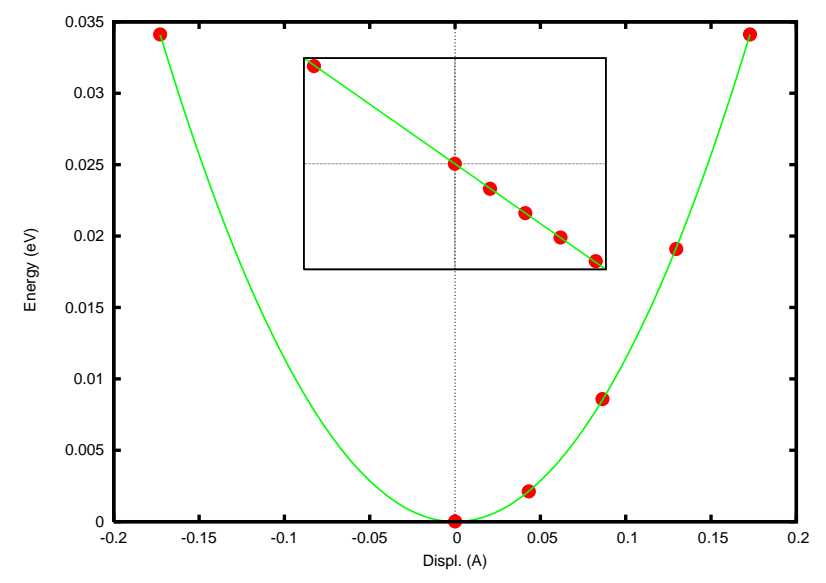

Figure 2: Frozen-phonon energy for a $E_{1 u}$ mode calculated by displacing Ca along the $x$ direction. The inset shows calculated forces acting upon $\mathrm{Ca}$ for the same displacements. The lines are, respectively, the least square quadratic and linear fits. The coefficients agree within $6 \%$ and yield for the phonon frequences of $120(123) \mathrm{cm}^{-1}$. (Color online)

Note that the mode is very harmonic. The calculated frequency is $123 \mathrm{~cm}^{-1}$ from the total energy fit, and 120 $\mathrm{cm}^{-1}$ from the forces, indicating a good convergence. This frequency is similar to the corresponding frequency

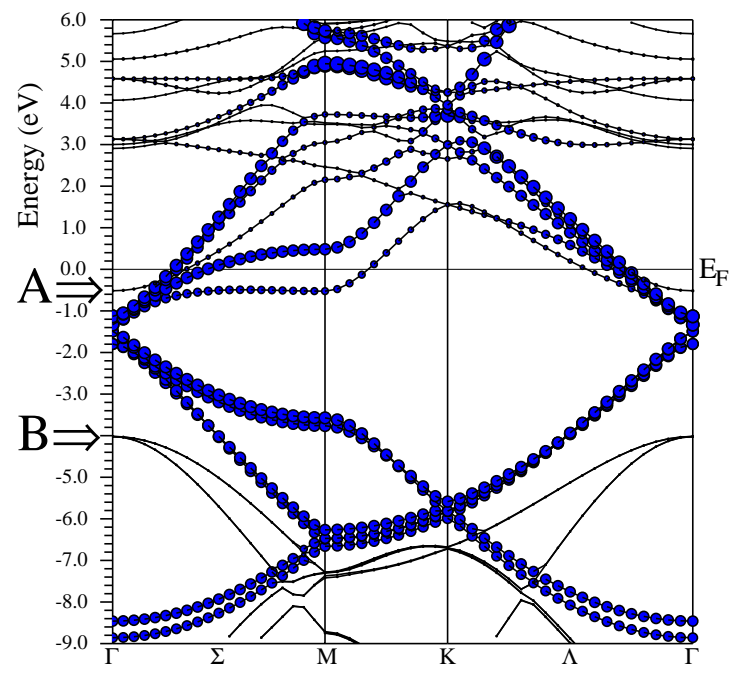

Figure 3: Band structure of the bilayer graphene $\mathrm{Ca}_{6} \mathrm{CaC}_{6}$. The size of the symbols indicates the relative $\mathrm{C}-\pi$ character of the electronic states. The arrow "A" points to interlayer nearly-free-electron $\zeta$ band (see text) and the arrow "B" to the bonding $\sigma$ band.(Color online).

in the bulk $\mathrm{CaC}_{6}[12], \approx 115 \mathrm{~cm}^{-1}$, supporting our conjecture that the phonon properties of a Ca layer sandwiched between two $\mathrm{C}$ layers are very similar to those of $\mathrm{Ca}$ in $\mathrm{CaC}_{6}$. This result was computed for purely $\mathrm{Ca}$ displacements; allowing for the weak hybridization with $\mathrm{C}$ mode of the same symmetry would bring the frequency slightly down, making it even closer to that in $\mathrm{CaC}_{6}[12]$. We have also calculated the two $A_{1 g}$ modes: the in-plane $\mathrm{C}$ mode, that is expectedly hard, $1370 \mathrm{~cm}^{-1}$, and the one that corresponds to breathing between the $\mathrm{C}$ planes. The latter mode is absent in the bulk compound. It appears to be very soft, $108 \mathrm{~cm}^{-1}$. Given that the position of the free-electron band strongly depends on the interlayer distance, we assume it will interact strongly with that band and thus open an additional electron-phonon coupling channel, absent in $\mathrm{CaC}_{6}$.

Let us now turn to the electronic properties. In Fig. 3 we show the calculated bands with $\mathrm{C} p_{z}$ character emphasized (the so-called $\pi$ bands). Obviously, the Dirac points are now well below the Fermi level. More importantly, we find the Ca-derived nearly free electron band crossing the Fermi level with its bottom located at the $\Gamma$ point $0.5 \mathrm{eV}$ below the Fermi level. If we place the intercalated bilayer graphene on the "Cambridge plot" from Ref. [10] we observe it to be located above $\mathrm{CaC}_{6}$, partially due to the nominally twice smaller doping, $1 / 6$ vs. $1 / 3$, see Fig. 4 .

Other characteristics of the calculated electronic structure are shown in Fig. 5 (density of states) and 6 (Fermi surface). Several observations are in order. First, the density of states per carbon at the Fermi level is very 


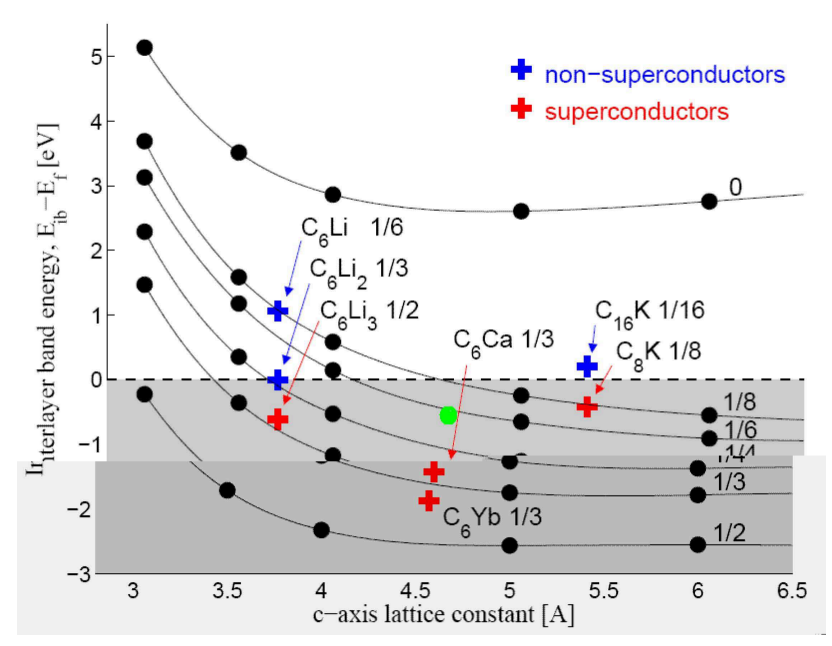

Figure 4: Correlation between the position of nearly free band, brought down to chemical potential due to $\mathrm{Ca}$ and superconducting properties of different materials, from [10]. We added to this figure a point (green circle) representing $\mathrm{C}_{6} \mathrm{CaC}_{6}$ (cf. Fig. 3).(Color Online).

similar to that of the bulk $\mathrm{CaC}_{6}: 2.5$ states per $\mathrm{C}_{12}$ and compared to 1.5 per $\mathrm{C}_{6}$ in $\mathrm{CaC}_{6}[11]$. Second, compared to $\mathrm{CaC}_{6}$ (see Ref. [11], Fig. 6), the Fermi level appears in a minimum of the density of states between two large peaks at $\sim \pm 0.5 \mathrm{eV}$, a feature favorable for crystal stability. Finally, it is worth noting that the Ca-projected DOS is at least half of the C-projected one. The former comes predominantly from the interlayer band. Since the LAPW program projects the DOS onto the muffintin spheres, and the interstitial space in this structure is huge, this indicates that the DOS of the interlayer band is comparable with that of the $\mathrm{C}-\pi$ bands, despite the fact that the corresponding Fermi surface (small orange circle around the $\Gamma$ point in Fig. (6) is so small compared to the Fermi surfaces of $\mathrm{CaC}_{6}$ and $\mathrm{YbC}_{6}$ (Refs. [6, 18]). This is a manifestation of the two-dimensional character of the band structure and independence of the DOS (and the corresponding electron-phonon coupling [19]) of the doping level. A useful, albeit elementary, excersise is to compare DOS of a 3D parabolic band at a given filling with that of a $2 \mathrm{D}$ parabolic band with the same mass. It is easy to see that the latter is larger as long as the Fermi vector in the 3D band $k_{F}<\pi / c$, where $c$ is the interlayer spacing in the 3D system (geometrical meaning: as long as the Fermi sphere diameter is smaller than the interplanar distance in the reciprocal space). Note that in $\mathrm{CaC}_{6}$ and $\mathrm{YbC}_{6}$ this condition is not satisfied, and therefore going from $3 \mathrm{D}$ to $2 \mathrm{D}$ is beneficial for superconductivity.

As discussed, the coupling of the interlayer band with phonons is essentially doping-independent (as shown in Ref. [19], this holds even when the FS is so small and the Kohn anomaly so strong that the phonon self-energy

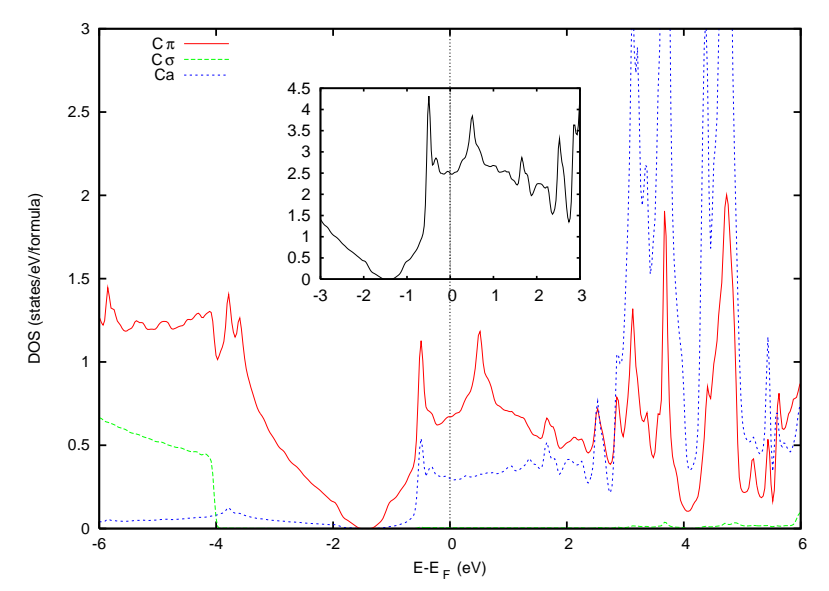

Figure 5: Density of states projected onto individual atomic functions. Note the sharp onset of the bonding $\mathrm{C} \sigma$ bands (long-dash green line) below $-4 \mathrm{eV}$ and the onset of the $\zeta$ band (short-dash blue line) above $-0.5 \mathrm{eV}$. The inset shows the total DOS, which is very close to that of the bulk $\mathrm{CaC}_{6}$ if compared on the per-C basis. (Color online).

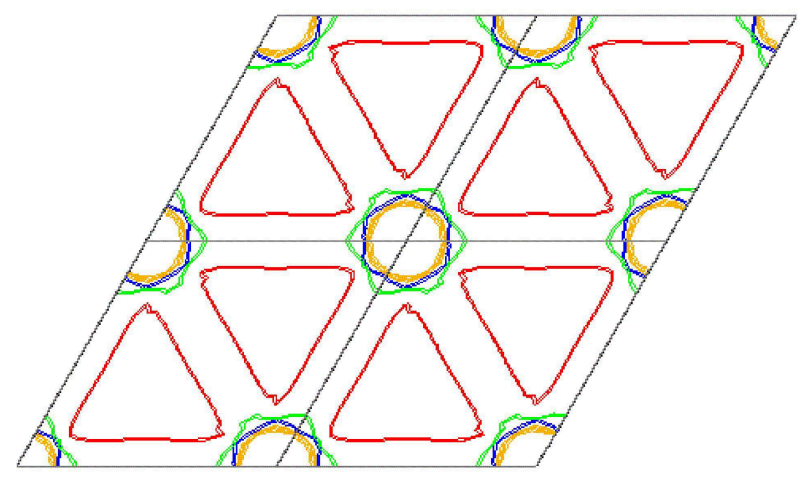

Figure 6: 2D Fermi surface of $\mathrm{C}_{6} \mathrm{CaC}_{6}$. Note a perfect circle around the $\Gamma$ point (orange online), derived from the $\zeta$ band, and the triangular Fermi contours derived from the Dirac electrons. (Color online).

should be calculated self-consistently including the feedback effects). Based on this, we suggest that pairing coupling constant in $\mathrm{Ca}_{6} \mathrm{CaC}_{6}$ is similar to the parent $3 \mathrm{D}$ compound. However, this does not mean that additional doping does not help in terms of enhancing the coupling. As we know from the $\mathrm{CaC}_{6}$ calculations, about half of the total coupling comes from the $\mathrm{C} \pi$ bands, and these, being strongly non-parabolic (a triangular shape of the largest Fermi surface in Fig. 6 6attests to the fact that the $\mathrm{C}$ bands are still fairly close to the Dirac dispersion), do show energy-dependent DOS, and, by implication, energy-dependent electron phonon coupling. Adding surface $\mathrm{Ca}$ atoms to the intercalated bilayer graphene has potential to further increase the coupling and enhance the critical temperature. 
Our observation about the nearly free electron band crossing the Fermi level (Figs. 344) and the similarity of the phonon spectra in the intercalated bilayer and in the bulk $\mathrm{CaC}_{6}$ are the main results of this Letter. Based on these similarities, we conjecture that $\mathrm{Ca}$ intercalated graphene bilayer is a superconductor.

A few comments are in place regarding the practicality of these materials. We know now that the single and the bilayer graphenes are electronically inhomogeneous, as was seen by scanning probes [20, 21]. Effects of charge inomogeneity on the superconducting state would need to be addressed in details if indeed superconductivity is observed in these materials. Variations of local charge density in bilayer graphene would play a role of nonmagnetic impurities. As such, they would be subject to the Anderson theorem and thus would not be pair-breaking for the isotropic or nearly-isotropic s-wave superconductivity, as it is believed to be the case in $\mathrm{CaC}_{6}[13]$ and presumably is in $\mathrm{C}_{6} \mathrm{CaC}_{6}$. Another important effect left outside of the scope of this letter is the role of substrate. We assumed that substrate effects would be the strongest for a single layer. One would need to model the substrate in a manner consistent with the DFT approach used here to address its role specifically.

In conclusion, we propose to search for superconductivity in a $\mathrm{Ca}$ intercalated graphene bilayer. Our estimates of the phonon frequencies and possible electronphonon coupling constant make this material a plausible candidate for superconductivity with $T_{c}$ in the range of few $\mathrm{K}$, and possibly above $10 \mathrm{~K}$. We start with an undoped bilayer graphene that is nonsuperconducting. $\mathrm{Ca}$ intercalation renders a band structure that is rather close to that of the bulk $\mathrm{CaC}_{6}$, a known superconductor with $T_{c}=11.5 K .[2,43,4,5]$. Frequencies of zone center phonons are very close to those in $\mathrm{CaC}_{6}$. The nearly free electron band crosses the Fermi level in $\mathrm{C}_{6} \mathrm{CaC}_{6}$, just as it does in all known superconducting intercalated graphites 10], thus satisfying the "Cambridge conjecture", Fig(4). Despite the lower doping level, the 2D character of this band provides an even higher density of states than in $\mathrm{CaC}_{6}$.

The path to design superconducting material proposed here is a bottom up approach, similar to the multilayer superconducting films. If the ideas presented here turn out to be relevant we might see a new approach to upwarard scaling superconducting materials. More broadly these ideas fall within the approach of materials by design, where we are trying to design materials that target particular function, in this case superconducting properties with highest $T_{c}$.

We are grateful to I. Lukyanchuk and T. Wehling for useful discussions. This work was supported by by US
DoE BES and LDRD at Los Alamos.

* Electronic address: avb@lanl.gov

[1] H.B. Heersche, P. Jarillo-Herrero, J.B. Oostinga, L.M.K. Vandersypen, and A.F. Morpurgo, Nature, bf 446, p 05555, (2007).

[2] I.T. Belash, O.V. Zharikov, and A.V. Palnichenko, Synt. Metals, 34, 455 (1989); ibid, 36, 283 (2002).

[3] T. E. Weller, M. Ellerby, S. S. Saxena, R. P. Smith, and N. T. Skipper, Nat. Phys. 1, 39 (2005).

[4] N. Emery, C. Hérold, M. d'Astuto, V. Garcia, C. Bellin, J. F. Marêché, P. Lagrange, and G. Loupias, Phys. Rev. Lett. 95, 087003 (2005).

[5] N. B. Hannay, T. H. Geballe, B. T. Matthias, K. Andres, P. Schmidt, and D. MacNair, Phys. Rev. Lett. 14, 225 (1965).

[6] I. I. Mazin, L. Boeri, O.V. Dolgov, A.A. Golubov, G.B. Bachelet, M. Giantomassi, and O.K.Andersen., Physica C460, 116 (2007).

[7] A. Novoselov et al., Nature 438, 197 (2005).

[8] Y.Zhang et al., Nature 438, 201 (2005).

[9] B. Uchoa and A. H. Castro Neto, Phys. Rev. Lett. 98, 146801 (2007); B. Uchoa, C.-Y. Lin, and A. H. Castro Neto, Phys. Rev. B 77, 035420, (2008).

[10] G. Csányi, P. B. Littlewood, A. H. Nevidomskyy, C. J. Pickard, and B. D. Simons, Nat. Phys. 1, 42 (2005)

[11] I.I. Mazin, Phys. Rev. Lett. 95, 227001, (2005).

[12] M. Calandra and F. Mauri, Phys. Rev. Lett. 95, 237002 (2005).

[13] A. Sanna, G. Profeta, A. Floris, A. Marini, E.K.U. Gross, S. Massidda, Phys. Rev. B 75, (2007) 020511(R).

[14] Recent measurements of the Ca isotope effect (D. G. Hinks, D. Rosenmann, H. Claus, M. S. Bailey, and J. D. Jorgensen, Phys. Rev. B 75, 014509, 2007) suggest that the Ca contribution may be even higher.

[15] One can deposit the $\mathrm{Ca}$ atoms on the single graphene sheet. However in this case effect of top Ca atoms will be very different and one would need a detail characterization of a substrate.

[16] It might also be possible to cover bilayer graphene with $\mathrm{Ca}$, adding carriers and likely improving superconducting properties, but the phonon properties of a Ca overlayer will be very different from that of of intercalated $\mathrm{Ca}$ and are beyond the scope of this Letter.

[17] P. Blaha et al., WIEN2k, An Augmented Plane Wave + Local Orbitals Program for Calculating Crystal Properties, Karlheinz Schwarz, Techn. Universität Wien, Austria, 2001.

[18] I. I. Mazin and S.L. Molodtsov, Phys.Rev. B72, 172504 (2005).

[19] O. V. Dolgov, O. K. Andersen, and I. I. Mazin, Phys. Rev. B 77, 014517 (2008).

[20] G. M. Rutter, J. N. Crain, N. P. Guisinger, T. Li, P. N. First and J. A. Stroscio, Science, 317, 219, (2007).

[21] J. Martin, N. Akerman, G. Ulbricht, T. Lohmann, J. H. Smet, K. von Klitzing and A. Yacoby, Nature Physics, 4, 144, (2008). 